\title{
Evaluation of Primary Stability of Immediate Implant Placement in Extraction Sockets in vitro
}

\section{Background and Aim}

Immediate implant placement and restoration following tooth extraction allow us to perform a minimally invasive surgery and shorten treatment periods. Primary stability is essential, as it keeps implants stable until osseointegration is gained. Sufficient primary stability has to be obtained with the only $4 \sim 5 \mathrm{~mm}$ at the apical part of implants, because it would be the part inserted into alveolar bone of extraction sockets (Fig.1).
Therefore, the apical design of implants is important for immediate placement.

The Aim of this research is to evaluate the primary stability of implants with different body designs in vitro. Two types of implants Straight type and Tapered type) were tested. The primary stability was assessed by the insertion torque when the apical part of implants was inserted into bone models.

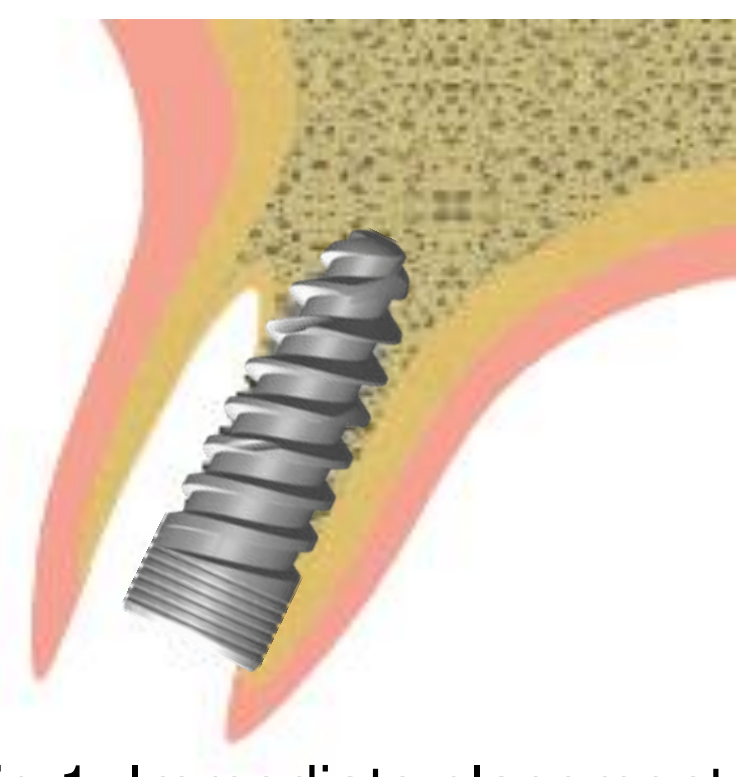

Fig.1: Immediate placement

\section{Methods and Materials}

\section{Methods}

Straight type implants (GC Implant Aadva Standard implant, GC) and Tapered type implants (GC Implant Aadva Tapered implant, GC) were inserted into a bone model with a vertical constant load of $500 \mathrm{~g}$ by using the insertion torque test machine (PROTEC Corporation) and insertion the insertion torque test machine (PROTEC Corporation) and insertion
torque was recorded (Fig.2, 3). The insertion torque at $5 \mathrm{~mm}$ insertion torque was recorded
depth was compared.

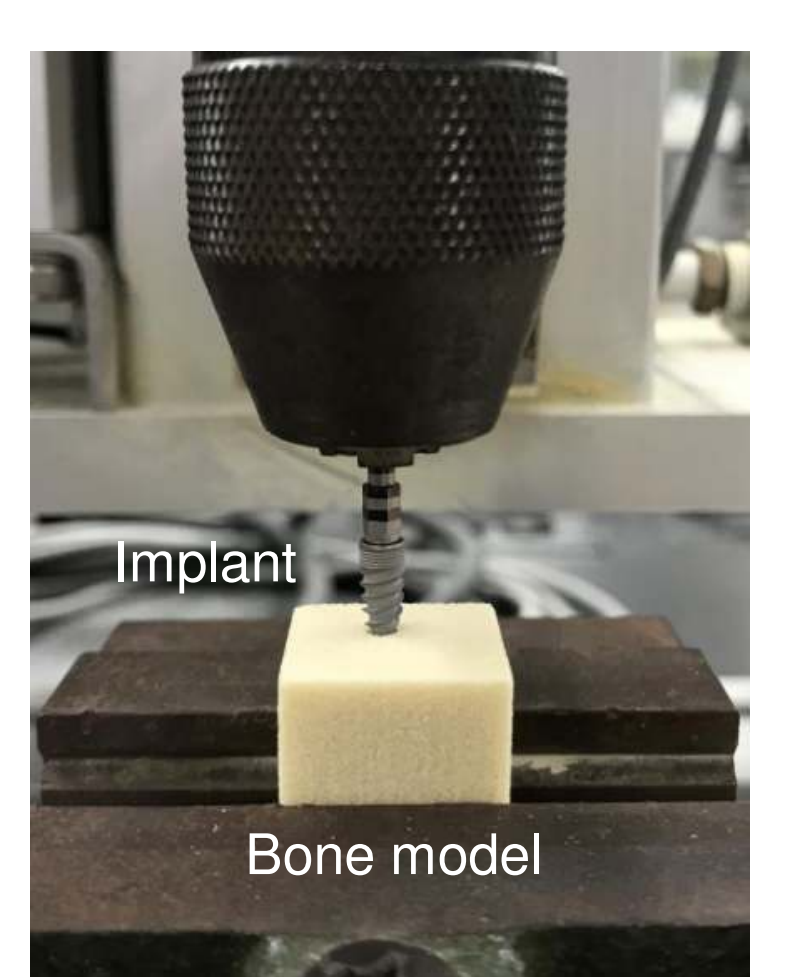

Fig.2: Insertion test

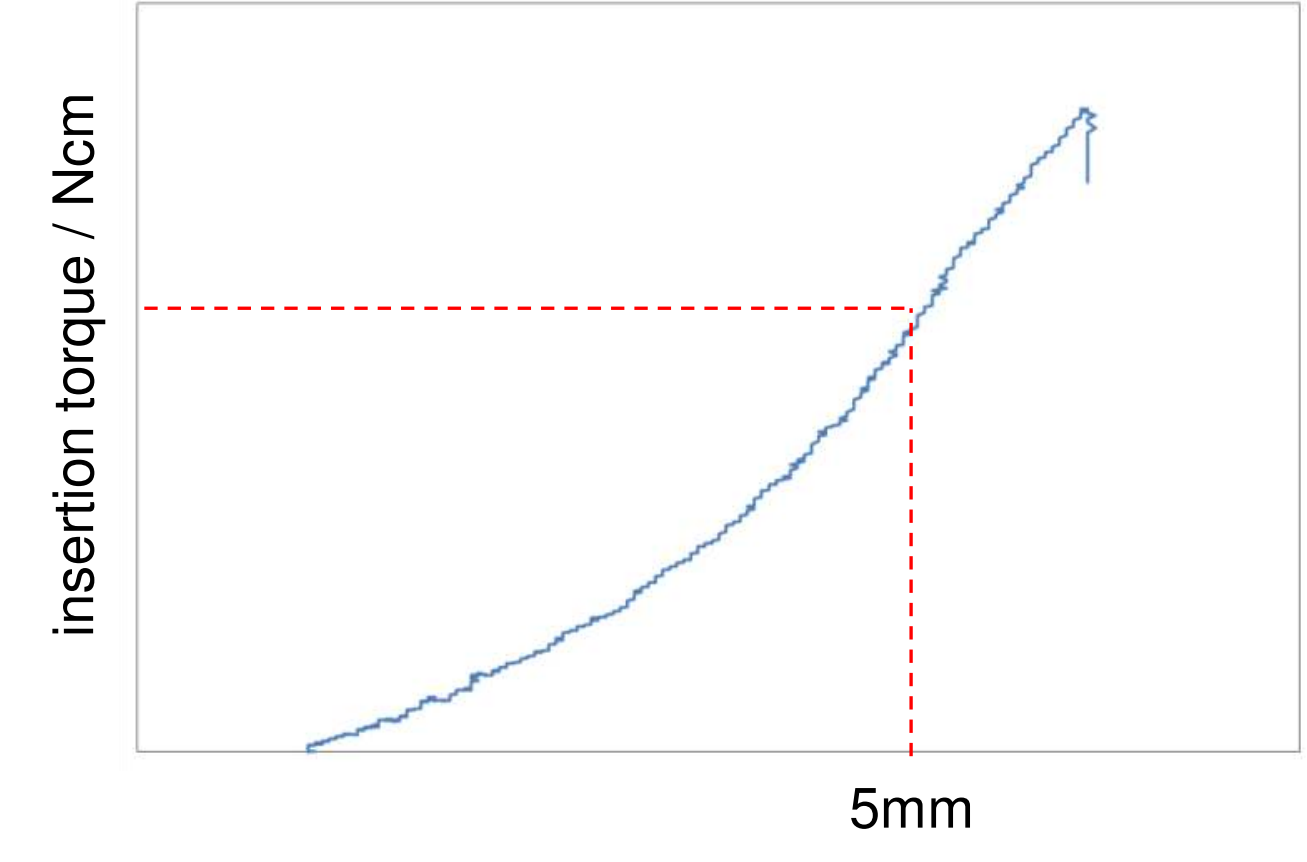

insertion depth / $\mathrm{mm}$

Fig.3: Insertion depth - torque

\section{Materials}

\section{Implants}

Following 6 types of implants were used.

Straight type : Narrow, Regular, Wide

Tapered type : Narrow, Regular, Wide

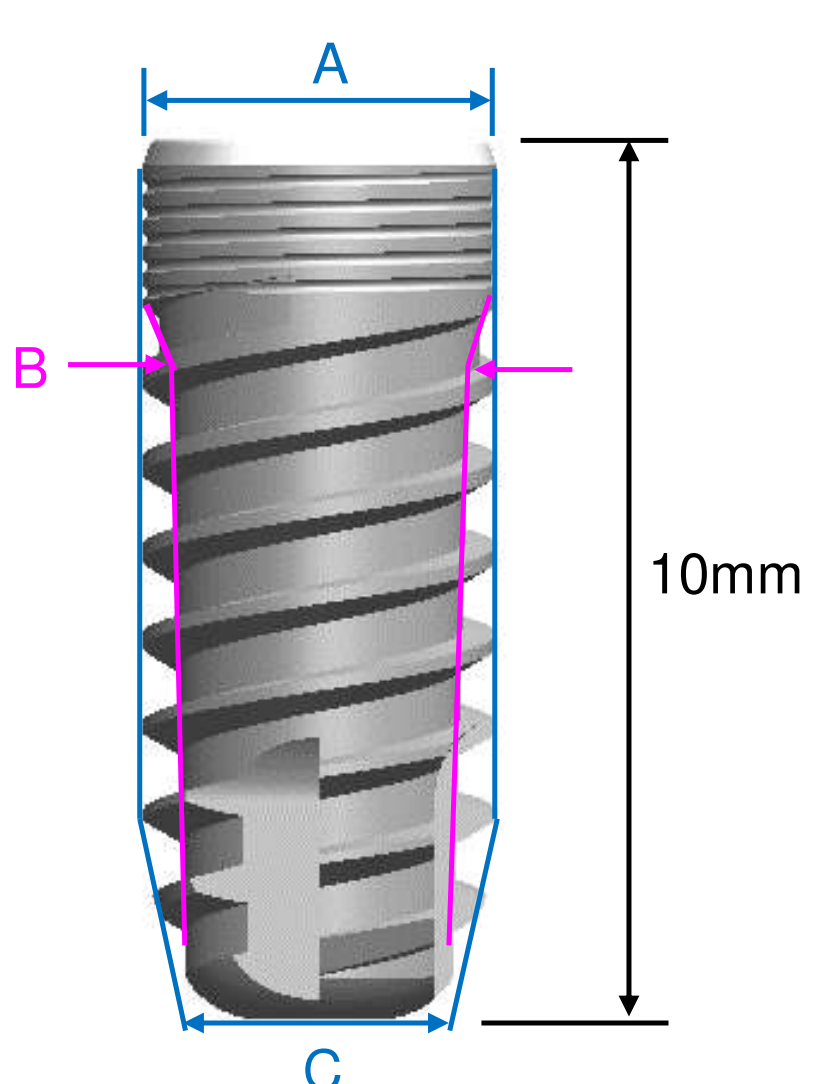

Straight type (ST)

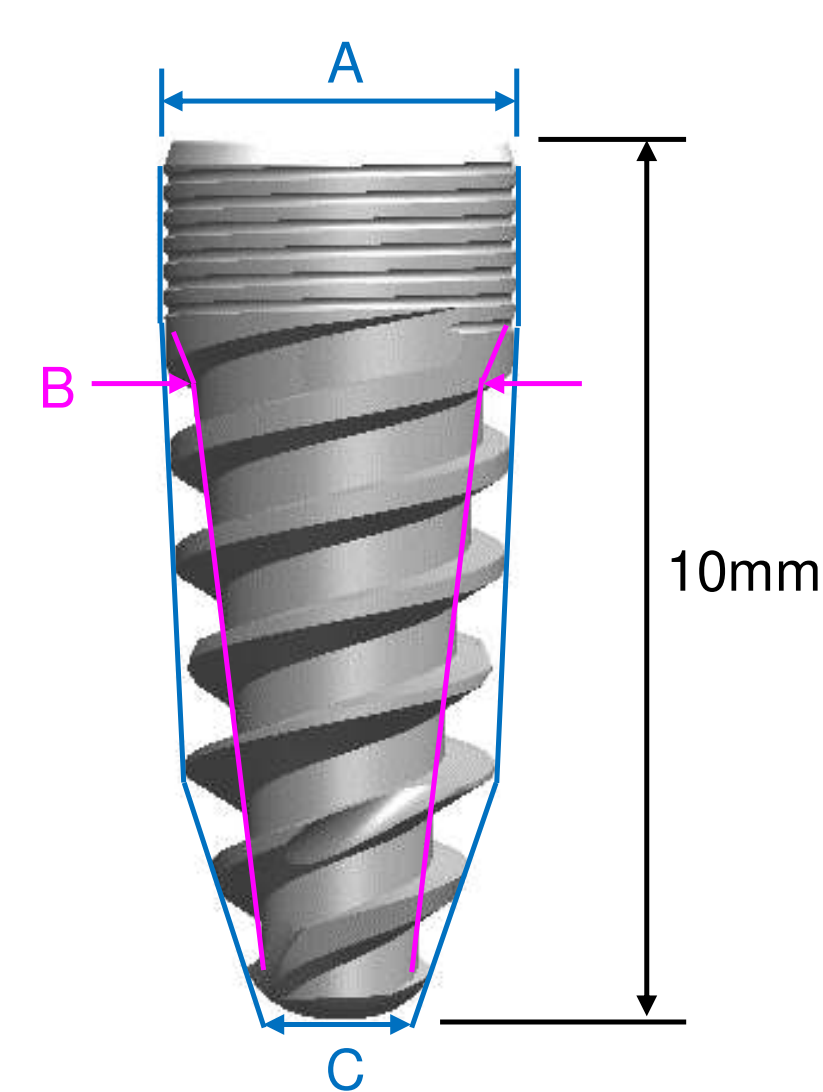

Tapered type (TP)

\begin{tabular}{|lcccccc|}
\hline & \multicolumn{2}{c}{ Narrow } & \multicolumn{2}{c}{ Regular } & \multicolumn{2}{c|}{ Wide } \\
\hline & ST & TP & ST & TP & ST & TP \\
\hline A & \multicolumn{2}{|c|}{3.3} & \multicolumn{2}{c}{4.0} & \multicolumn{2}{c|}{5.0} \\
\hline B & \multicolumn{2}{|c}{2.7} & \multicolumn{2}{c}{3.4} & \multicolumn{2}{c}{4.4} \\
\hline C & 2.0 & 1.3 & 2.7 & 1.8 & 3.7 & 2.8 \\
\hline
\end{tabular}

Fig.4: Aadva Implants

\section{Bone models}

Polyurethane foam blocks (SAWBONE, Pacific Research Laboratories) with density of 30 pcf were used.

Drills

The minimum diameters of twist drills which were sufficient for insertion of each implants were selected from following 7 sizes (Fig.5). The diameters of drill holes in this test are shown in Fig.6. The drilling depth was $9 \mathrm{~mm}$. It was deep enough to insert implants by $5 \mathrm{~mm}$.

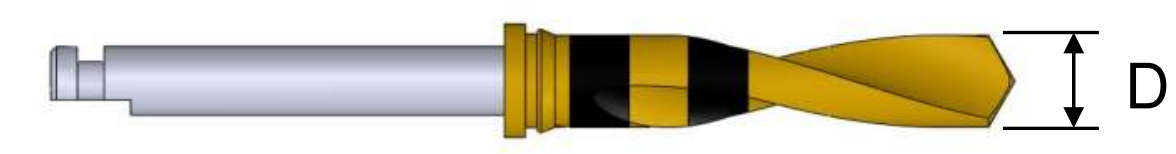

$\mathrm{D}: \phi 2.0,2.7,2.9,3.1,3.4,3.6,3.9 \mathrm{~mm}$

Fig.5: Twist drills

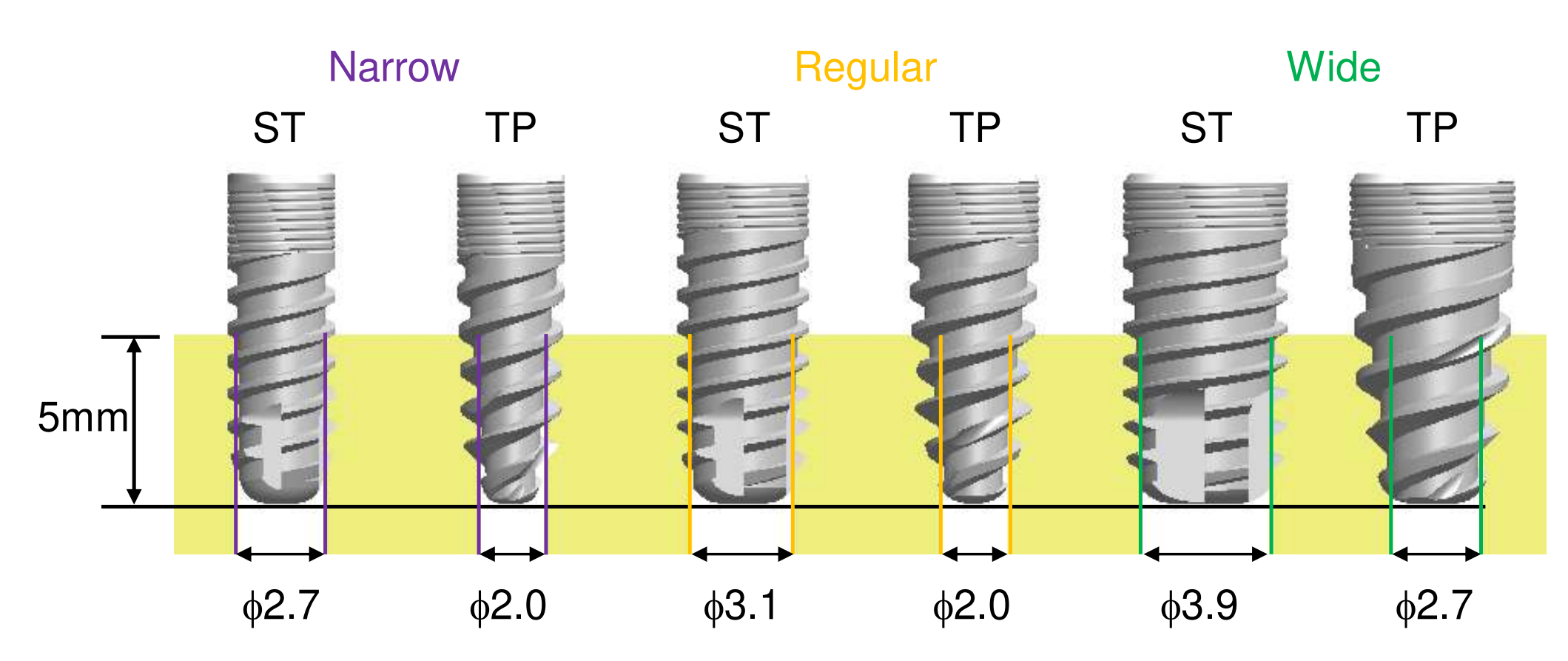

Fig.6: Relationship between implant body and drill hole

\section{Results}

Insertion torque for an insertion depth of $5 \mathrm{~mm}$ is shown in Fig.7.

Tapered implants could be inserted with using narrower size drills and had tendency to show larger insertion torque compared with straight implants.

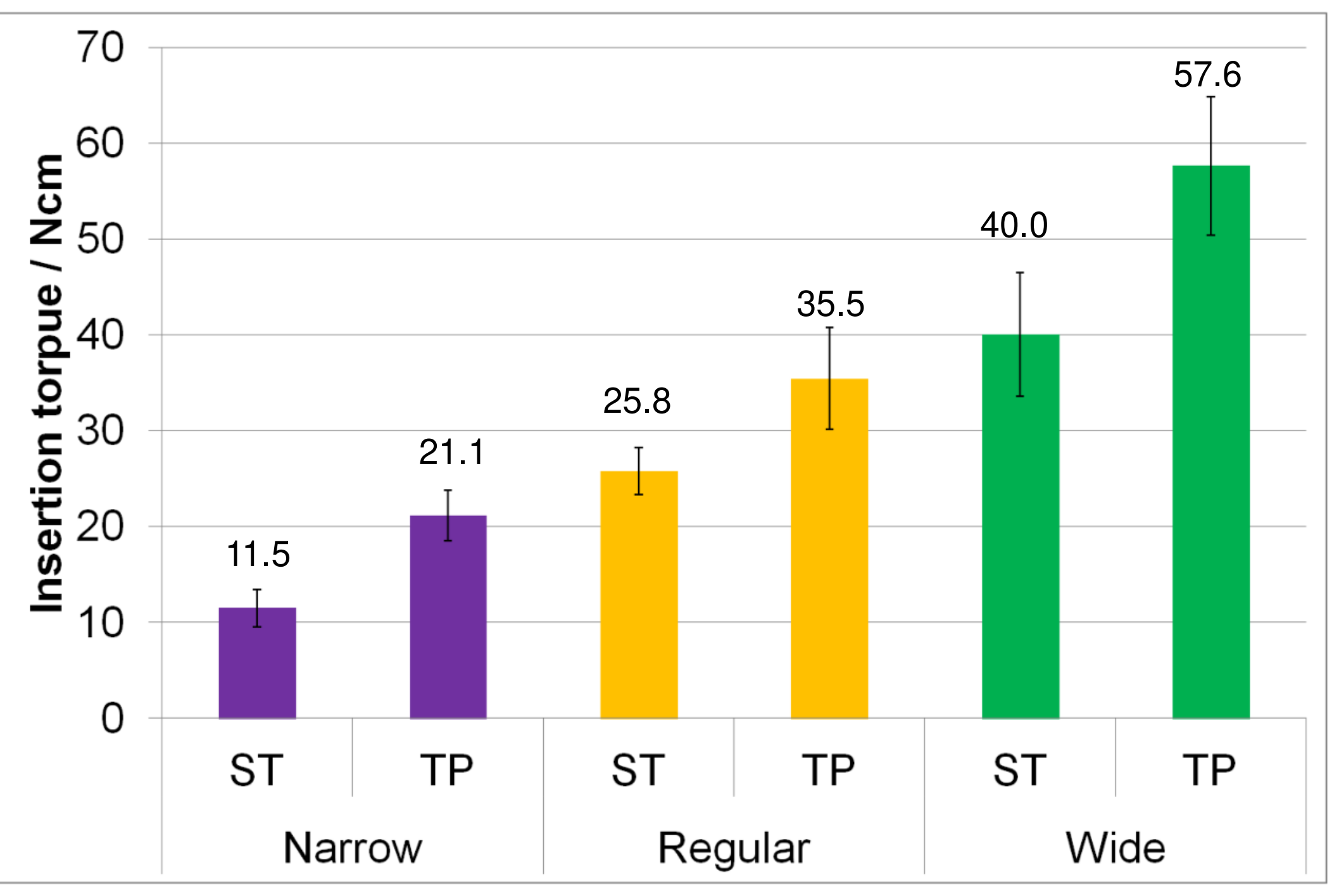

Fig.7: Insertion torque of each implants $(n=3)$

\section{Conclusions}

Compared to Straight implants, Tapered implants can be screwed into narrower drill holes because of its narrower apical end and advance into bone, expanding the hole with its tapered body (Fig.5). This feature seems to have led to sufficient insertion torque at the depth of only $4 \sim 5 \mathrm{~mm}$. However, it should be noted that wide implants can show very high torque. In this case, a larger diameter drill should be used.

These results suggested that the tapered implants can be used with good primary stability even in cases of immediate placement.
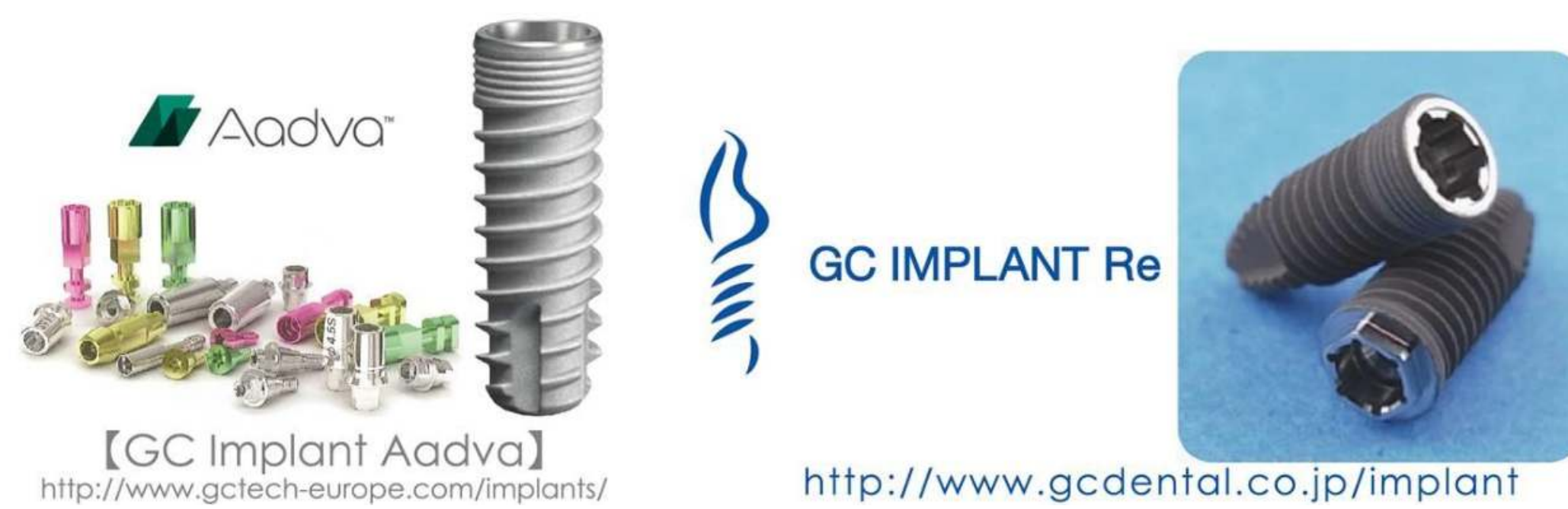

http://www.gcdental.co.jp/implant 